\title{
Estudo de Fase II com Doses Fracionadas Diárias de Etoposide Oral em Pacientes com Câncer de Mama Estádio IV Refratário a Múltiplos Esquemas Quimioterápicos
}

\author{
Phase II Study of Fractionated Daily Doses of Oral Etoposide in Patients With \\ Stage IV Breast Cancer Refractory to Multiple Chemotherapy Regimens
}

\begin{abstract}
Fábio Silva Leal ${ }^{1,2}$, Flávio Silva Brandão ${ }^{1,3}$, Romeu Roberto Rigby ${ }^{1,3}$, Gisele Marinho dos Santos ${ }^{1,3}$, Antonio José Esteves Bugarin ${ }^{1,3}$, Flávia Cupello Tamiozzo ${ }^{1,3}$, Mônica Luciana Agostinho Padoan ${ }^{1,3}$, Giovana Kovaleski ${ }^{4}$, Gilberto Schwartsmann ${ }^{5}$, Hiram Silveira Lucas ${ }^{6}$
\end{abstract}

\section{Resumo}

O etoposide é um inibidor da topoisomerase II capaz de produzir respostas objetivas em cerca de $10 \%$ das pacientes com câncer de mama metastático após a falha a esquemas convencionais. A administração oral de doses fracionadas de etoposide produz um aumento significativo do tempo de exposição dos tecidos em níveis terapêuticos deste agente, aumentando o seu índice terapêutico. Neste artigo, são descritos os resultados de um ensaio clínico de fase II com etoposide oral em doses fracionadas diárias em 20 mulheres com câncer de mama metastático refratário a múltiplos esquemas quimioterápicos. Foram elegíveis pacientes entre 18-75 anos de idade, desempenho clínico entre 0-2 (ECOG), diagnóstico histopatológico de câncer de mama, doença metastática visceral, sem disfunção de órgãos vitais e sem envolvimento do Sistema Nervoso Central (SNC). A assinatura de um documento de consentimento pela paciente, segundo as normas da CONEP e do Comitê de Ética da Instituição, era condição necessária para inclusão. O conteúdo da ampola de etoposide para uso endovenoso foi administrado por via oral na dose de $20 \mathrm{mg} / \mathrm{m} 2$ a cada oito horas, diluído em veículo ácido (suco de laranja ou uva), diariamente por 14 dias, seguido de sete dias de intervalo. A cada 21 dias, as pacientes foram reavaliadas quanto à toxicidade (critério do NCI-CTC) e, a cada dois ciclos (42 dias), quanto à resposta tumoral (critério da RECIST). As pacientes foram tratadas até a progressão, toxicidade limitante ou desejo próprio de interromper o tratamento. Foram incluídas 20 pacientes no estudo, tendo sido analisado um total de 55 ciclos de tratamento, com uma mediana de dois ciclos por paciente (1-10). Os efeitos adversos mais observados foram: náusea (36\%), vômitos (24\%), mucosite (16\%) e neutropenia (14\%). Neutropenia febril ocorreu em apenas um caso (2\%). Não foram documentadas respostas objetivas. Entretanto, 9 pacientes apresentaram doença estável (45\%), algumas com duração prolongada (30+, 21+ e 18 semanas). A mediana de duração destas estabilizações de doença foi de 15 semanas (9-30+). Em conclusão, este esquema oral fracionado de etoposide foi bem tolerado, produzindo estabilizaçôes de doença duradouras, mas sem vantagem em termos de respostas objetivas, se comparado à sua forma de administração por via oral em dose única diária.

Palavras-chave: Etoposídeo; Neoplasias da mama; Metástase neoplásica

${ }^{1}$ Oncologista Clínico

${ }^{2}$ Chefe do Serviço de Oncologia do Hospital Mário Kröeff

${ }^{3}$ Serviço de Oncologia do Hospital Mário Kröeff

${ }^{4}$ Bióloga e Coordenadora de Pesquisa Clínica do Hospital Mário Kröeff

${ }^{5}$ Professor Titular da Universidade Federal do Rio Grande do Sul (UFRGS)

${ }^{6}$ Professor da Fundação Carlos Chagas Filho de Amparo à Pesquisa do Estado do Rio de Janeiro

Endereço para correspondência: Fábio Silva Leal. Serviço de Oncologia. Hospital Mario Kröeff - Rua Magé 326 - Penha Circular - Rio de Janeiro (RJ),

Brasil - CEP: 21020-130. E-mail: oncoleal@hotmail.com 


\section{INTRODUÇÃO}

Mulheres com câncer de mama metastático, cuja neoplasia seja receptor hormonal negativo, acometa não apenas partes moles e esqueleto, apresente progressão visceral e/ou seja receptor hormonal positivo, mas refratária aos tratamentos hormonais disponíveis, são consideradas candidatas ao tratamento quimioterápico paliativo $^{1,2}$.

Vários esquemas têm sido utilizados no tratamento quimioterápico dessas pacientes, principalmente sob a forma de combinaçôes de dois ou três agentes, como, por exemplo, a ciclofosfamida, as antraciclinas (doxorrubicina e epirrubicina), os taxanos (paclitaxel e docetaxel), fluoropirimidinas (5-fluorouracil, capecitabina e UFT), o metotrexate e outros ${ }^{2,3}$.

As combinações de agentes parecem oferecer maiores percentuais de respostas objetivas, maior duração de resposta e uma vantagem em sobrevida livre de progressão, quando comparadas com o uso de agentes únicos ${ }^{2,4}$. Essa potencial vantagem terapêutica do uso de combinações versus agentes únicos sequenciais deve sempre levar em conta a maior probabilidade de toxicidade e a modesta vantagem na sobrevida global das pacientes ${ }^{1,4,5}$.

Vários agentes têm sido estudados em pacientes com doença metastática, em progressão após o uso de esquemas de primeira ou segunda-linha, contendo os agentes acima mencionados. São exemplos a cisplatina, a carboplatina, a vimblastina, a mitomicina C, a gemcitabina, as modificações na forma de administração e no tipo de fluoropirimidinas e outros ${ }^{2,5,6}$. Os percentuais de resposta com esses agentes oscilam em torno de $10-20 \%$, com uma duração mediana de resposta de cerca de 3-6 meses ${ }^{1,6,7,8}$.

A ixabepilona, um análogo da epotilona $\mathrm{B}$, pode produzir respostas objetivas em cerca de $10 \%$ dos casos, com uma mediana de duração de seis meses e sobrevida global de cerca de oito meses?. Quando utilizada em segunda linha na doença metastática, a adição de bevacizumabe, um anticorpo antiVEGF, ao paclitaxel parece aumentar a duração das respostas em cerca de cinco meses, contudo sem vantagem na sobrevida global $^{10}$.

O uso de trastuzumabe, um anticorpo anti-HER-2, isoladamente ou em combinação com paclitaxel, pode também produzir respostas objetivas em pacientes HER-2 positivo ${ }^{11}$. Mais recentemente, a combinação de capecitabina ao agente lapatinibe foi associada a um ganho de cerca de quatro meses no tempo livre de progressão, quando comparado ao uso de capecitabina como agente único ${ }^{12}$.
O etoposide é um inibidor da enzima topoisomerase II e o seu efeito antitumoral in vitro é dependente do tempo de exposição celular a este agente. Estudos sugerem que os níveis plasmáticos de etoposide necessários para que se produza um efeito antitumoral em células tumorais in vitro sejam inferiores àqueles associados ao surgimento de toxicidade na medula óssea ${ }^{13}$.

Portanto, existe uma base racional para que sejam exploradas formas alternativas de administração de etoposide, as quais possam produzir níveis plasmáticos terapêuticos mais prolongados desse agente, mas inferiores às concentrações associadas a um maior risco de mielotoxicidade ${ }^{14}$.

Estudos pioneiros em pacientes com câncer de pulmão do tipo pequenas células sugerem que o uso oral continuado de etoposide produz um significativo aumento nos índices de resposta tumoral, quando comparados à sua administração endovenosa em infusão de uma hora por 3-5 dias ${ }^{13,14,15}$. Em pacientes com câncer de mama metastático refratário a esquemas de primeira linha, dados compilados de estudos de fase II com etoposide pela via intravenosa sugerem percentuais de resposta de cerca de $8 \%(0-14 \%)$, enquanto que o seu uso oral diário contínuo por 2-3 semanas está associado a respostas objetivas em cerca de $19 \%$ dos casos (0$35 \%)^{15,16,17}$.

Nosso grupo de pesquisa tem estudado a segurança e a eficácia do uso de etoposide em doses orais fracionadas diárias em tumores sólidos refratários, visando a otimizar o seu uso clínico dentro dos preceitos farmacológicos e farmacodinâmicos acima mencionados ${ }^{18}$. Em uma série de pacientes com Sarcoma de Kaposi associado à SIDA, essa estratégia foi capaz de atender satisfatoriamente a estes aspectos, levando a níveis terapêuticos mais prolongados no plasma dos pacientes, o que se traduziu em um maior índice de respostas tumorais e uma toxicidade manejável ${ }^{19,20}$.

Neste artigo, foi descrita a experiência com o uso de etoposide oral em doses fracionadas diárias em uma população de pacientes com câncer de mama metastático, as quais apresentaram progressão da doença após a administração de pelo menos dois esquemas de combinaçōes quimioterápicas.

\section{PACIENTES E MÉTODOS}

Neste estudo de fase II, foram consideradas para inclusão: mulheres com diagnóstico histopatológico comprovado de câncer de mama, com doença metastática em progressão visceral, não visceral ou ambas, com evidência de progressão clínica após pelo menos dois esquemas de tratamento quimioterápico paliativo. 
Esses esquemas incluíram antraciclinas para tratamento de primeira linha para a doença metastática (por exemplo, ciclofosfamida/doxorrubicina ou ciclofosfamida/ doxorrubicina/5-fluorouracil) e taxanos como tratamento de segunda linha (por exemplo, paclitaxel droga única). As pacientes receberam adicionalmente drogas em terceira linha, como vinorelbina, gemcitabina, mitomicina, vimblastina e outras. Hormonioterapia com tamoxifeno foi utilizada em alguns casos após o tratamento quimioterápico adjuvante.

Independentemente do estado de expressão de HER-2 no tumor, nenhuma das pacientes recebeu trastuzumab previamente ou posteriormente ao ingresso no estudo, devido às limitaçōes de acesso a este medicamento nesta Instituição.

As pacientes elegíveis deveriam ter entre 18-75 anos de idade, com desempenho clínico entre 0-2 (ECOG), função hepática normal ou até duas vezes os valores laboratoriais normais no caso de envolvimento metastático, funçôes renal, cardíaca, neurológica (sem evidências de metástases cerebrais) e mental preservadas. A assinatura de um documento de consentimento pela paciente, segundo as normas da CONEP e do Comitê de Ética da instituição, era condição necessária para inclusão no estudo.

As pacientes foram previamente submetidos a estadiamento através de exame clínico completo, exames laboratoriais e de imagem, incluindo Raios X de tórax, ultrassonografia do abdômen e cintilografia de esqueleto. A tomografia computadorizada foi realizada quando indicada pelo médico responsável. As análises de expressão de HER-2 e p53 no tumor foram realizadas pela técnica de imunoperoxidase, sendo que, na maioria dos casos, os exames foram realizados no Laboratório de Patologia da Santa Casa de Misericórdia do Rio de Janeiro.

O etoposide foi administrado por via oral na dose de $20 \mathrm{mg} / \mathrm{m}^{2}$ a cada 8 horas, diluído em veículo ácido (suco de laranja ou uva), diariamente por 14 dias, seguido de uma semana de repouso. Após cada ciclo de 21 dias, as pacientes foram reavaliadas quanto à toxicidade (critério do NCI-CTC), enquanto que a avaliação de resposta (critério da RECIST) foi realizada a cada dois ciclos de tratamento. As pacientes foram tratadas até a progressão, toxicidade limitante ou desejo próprio de interromper o tratamento. Neste estudo, não foi utilizado nenhum instrumento de avaliação objetiva de qualidade de vida.

\section{RESULTADOS}

\section{PACIENTES}

A inclusão de pacientes se deu a partir de Junho de 2006, tendo sido completada até Julho de 2007. Nesta população de 20 mulheres com câncer de mama metastático, a mediana de idade foi de 57 anos (28-74), com uma mediana de desempenho clínico de 1 (0-3). A mediana de regimes de tratamento quimioterápico prévios foi de 3 (2-6), sendo que todos as pacientes apresentavam progressão visceral (100\%).

Em 8 casos (40\%), os receptores hormonais (ER/ PR) eram ambos negativos; em 3 casos (15\%), os receptores eram ambos positivos; enquanto que, em 7 casos (35\%), estes eram positivo/negativo ou vice-versa. Em 2 casos (10\%), o estado dos receptores era desconhecido. Em 11 pacientes (55\%), a coloração pela técnica de imunoperoxidase para o $\mathrm{C}$-erb-B2 foi reagente positiva em 16 casos $(80 \%)$, enquanto a proteína p53 foi positiva no tumor primário em 11 casos (55\%). Estas características estão resumidas na Tabela 1.

\section{EFEITOS ADVERSOS DO TRATAMENTO}

Foi analisado um total de 55 ciclos de tratamento, com uma mediana de dois ciclos por paciente (1-10). Não foram documentados casos de redução de dose durante o tratamento. Entre os efeitos adversos não hematológicos documentados nessa população de pacientes, foram observados: náusea em 21 (36\%) ciclos administrados (13 em grau 1 e 8 em grau 2), vômitos em $13(24 \%)$ ciclos (9 em grau 1, 3 em grau 2 e 1 em grau 3), diarreia em 3 (5\%) ciclos (1 em grau 1 e 2 em grau 2), mucosite em 9 (16\%) dos ciclos (4 em grau $1 \mathrm{e}$ 5 em grau 2). Ocorreram episódios isolados de taquicardia, tonturas e eritema cutâneo, todos reversíveis e sem repercussão clínica significativa. Estes resultados estão apresentados na Tabela 2.

Os efeitos adversos hematológicos consistiram de anemia em 24 (44\%) ciclos administrados (2 em grau 1, 21 em grau 2 e 1 em grau 3), neutropenia em 8 (14\%) ciclos (4 em grau 2, 3 em grau 3 e 1 em grau 4), enquanto que trombocitopenia ocorreu em $1(2 \%)$ ciclo em grau 4. Febre foi observada em 3 (6\%) dos casos, com quadro de neutropenia febril e infecção documentada em apenas 1 caso (2\%). Estes resultados estão apresentados na Tabela 3.

\section{ATIVIDADE ANTITUMORAL}

Não foram documentadas respostas objetivas, segundo critérios da Response Evaluation Criteria in Solid Tumors (RECIST). Entretanto, 9 pacientes apresentaram doença estável (45\%), algumas com duração prolongada $(30+, 21+$ e 18 semanas). A mediana de duração destas estabilizações de doença foi de 15 semanas (9-30+). Nos demais 11 casos $(55 \%)$, houve progressão da doença. Esses resultados estão resumidos nas Tabelas 4 e 5. 
Tabela 1. Características das pacientes com câncer de mama metastático incluídas no estudo de fase II com etoposide oral em doses fracionadas diárias $(n=20)$

\begin{tabular}{|c|c|}
\hline Idade (anos; mediana) & $57(28-74)$ \\
\hline Desempenho clínico (ECOG; mediana) & $1(0-3)$ \\
\hline Pacientes com doença visceral (\%) & $20(100)$ \\
\hline Fígado isolado (\%) & 7 (35) \\
\hline Pulmão isolado (\%) & $315)$ \\
\hline Múltiplos sítios (\%) & $10(50)$ \\
\hline \multicolumn{2}{|l|}{ Receptores hormonais: } \\
\hline RE/RP -/- (\%) & $8(40)$ \\
\hline $\mathrm{RE} / \mathrm{RP}+/+(\%)$ & $315)$ \\
\hline RE/RP +/- ou -/+ (\%) & $7 \quad(35)$ \\
\hline RE/RP desconhecidos (\%) & $2(10)$ \\
\hline \multicolumn{2}{|l|}{ C-ERB-B2: } \\
\hline Positivo & $16(80)$ \\
\hline Negativo & $4(20)$ \\
\hline Esquemas de QT prévios (mediana) & $3(2-6)$ \\
\hline
\end{tabular}

Abreviaturas: Eastern Cooperative Oncology Group Performance Status criteria (ECOG); receptores estrogênicos (RE); receptores de progesterona (RP); receptor do fator de crescimento epidérmico-2

(C-ERB-b2); quimioterapia (QT)

Tabela 2. Perfil de toxicidade não hematológica das pacientes incluídas no estudo de fase Il com etoposide oral em doses fracionadas diárias $(\mathrm{n}=20)$

\begin{tabular}{|c|c|c|c|c|}
\hline Total de ciclos/pacientes avaliadas & \multicolumn{4}{|c|}{55} \\
\hline Número de ciclos/paciente (mediana) & \multicolumn{4}{|c|}{$2(1-10)$} \\
\hline \multirow{2}{*}{ Tipo de toxicidade } & \multicolumn{4}{|c|}{ Grau (NCl-CTC; \%) } \\
\hline & 1 & 2 & 3 & 4 \\
\hline Náusea & $13(23,6)$ & $8(14,5)$ & - & - \\
\hline Vômitos & $9(16,3)$ & $3(5,4)$ & $1(1,8)$ & - \\
\hline Diarreia & $1 \quad(1,8)$ & $2(3,6)$ & - & - \\
\hline Mucosite & $4 \quad(7,2)$ & $5(9,1)$ & - & - \\
\hline Febre & - & $1(1,8)$ & - & - \\
\hline Tonturas & $3(5,4)$ & - & - & - \\
\hline Taquicardia & $1 \quad(1,8)$ & - & - & - \\
\hline
\end{tabular}

Abreviatura: National Cancer Institute Common Toxicity Criteria (NCI-CTC)

Tabela 3. Perfil de toxicidade hematológica das pacientes incluídas no estudo de fase II com etoposide oral em doses fracionadas diárias $(n=20)$

\begin{tabular}{|c|c|c|c|c|}
\hline Total de ciclos/pacientes avaliadas & \multicolumn{4}{|c|}{55} \\
\hline Número de ciclos/paciente (mediana) & \multicolumn{4}{|c|}{$2(1-10)$} \\
\hline \multirow{2}{*}{ Tipo de toxicidade } & \multicolumn{4}{|c|}{ Grau (NCl-CTC; \%) } \\
\hline & 1 & 2 & 3 & 4 \\
\hline Anemia & $2(3,6)$ & $21(38,2)$ & $1(1,8)$ & - \\
\hline Neutropenia & - & $4(7,3)$ & $3(5,5)$ & $1(1,8)$ \\
\hline Trombocitopenia & - & - & - & $1(1,8)$ \\
\hline Neutropenia febril & - & - & $1(1,8)$ & - \\
\hline
\end{tabular}

Abreviatura: National Cancer Institute Common Toxicity Criteria (NCI-CTC) 
Tabela 4. Atividade Antitumoral observada em pacientes incluídas no estudo de fase II com etoposide oral em doses fracionadas diárias $(n=20)$

\begin{tabular}{c|c}
\hline $\begin{array}{c}\text { Tipos de Resposta } \\
\text { (RECIST) }\end{array}$ & $\begin{array}{c}\text { Número de Pacientes } \\
\text { (\%) }\end{array}$ \\
\hline Resposta Completa & $-(0)$ \\
\hline Resposta Parcial & $-(0)$ \\
\hline Estabilização de Doença & $9(45)$ \\
\hline Progressão de Doença & $11(55)$ \\
\hline
\end{tabular}

Abreviatura: Response Evaluation Criteria in Solid Tumors (RECIST)

Tabela 5. Duração das estabilizações de doença (RECIST) observadas em pacientes incluídas no estudo de fase II com etoposide oral em doses fracionadas diárias $(n=9)$

\begin{tabular}{c|c}
\hline $\begin{array}{c}\text { Pacientes (\# de } \\
\text { registro no estudo) }\end{array}$ & $\begin{array}{c}\text { Tempo de Estabilização } \\
\text { (semanas) }\end{array}$ \\
\hline 3 & 12 \\
\hline 4 & 12 \\
\hline 5 & 15 \\
\hline 7 & 12 \\
\hline 8 & 9 \\
\hline 9 & $30+$ \\
\hline 10 & 18 \\
\hline 11 & 15 \\
\hline 15 & $21+$ \\
\hline $\begin{array}{c}\text { Mediana de duração } \\
\text { (semanas) }\end{array}$ & $15(9-30+)$ \\
\hline
\end{tabular}

Abreviaturas: Response Evaluation Criteria in Solid Tumors (RECIST)

\section{DISCUSSÃO}

As pacientes com câncer de mama metastático em progressão clínica após falha a 2-3 esquemas de tratamento quimioterápico têm um prognóstico muito reservado. Via de regra, a mediana de sobrevida nesses casos é inferior a 3-6 meses ${ }^{1,3}$. Em muitas instituições, pacientes nessas condições são tratadas apenas sintomaticamente, ou, no caso de apresentarem desempenho clínico entre 0-2, podem ser incluídas em protocolos experimentais com novas drogas ou abordagens $s^{1,2,3}$.

Considerando a necessidade de novas opções terapêuticas para esta população de pacientes, o objetivo no presente estudo foi explorar ao máximo as informaçôes disponíveis sobre o agente etoposide na literatura, quanto à sua forma de administração, mecanismo de ação, farmacocinética no plasma e efeitos farmacodinâmicos ${ }^{15}$ 18,19.

Estudos anteriores publicados por este grupo demonstraram que a administração oral de doses fracionadas de etoposide produz um aumento significativo do tempo de exposição dos tecidos às doses terapêuticas deste agente ${ }^{18,19,20}$. Através desta estratégia, concentraçōes ativas deste agente foram mantidas no plasma por cerca de 4 horas após cada administração. Isto se refletiu em um ganho significativo nos índices de respostas em pacientes com Sarcoma de Kaposi associado à SIDA ${ }^{18,19}$.

O padrão e severidade dos efeitos adversos do etoposide administrado em doses fracionadas neste estudo foram considerados toleráveis, com raros episódios de sépsis ou leucopenia febril, e a ausência de letalidade induzida pelo tratamento. Náusea, vômitos, mucosite e diarreia foram documentadas, mas com frequência e severidade comparáveis à relatada na literatura com o uso de etoposide oral em dose única diária ${ }^{19}$.

Infelizmente, os índices de respostas, documentados nas pacientes com câncer de mama metastático, incluídos nesta amostra, não foram claramente superiores àqueles descritos na literatura em sua administração oral diária em dose única ${ }^{15,16,17}$. Entretanto, a observação de $45 \%$ de casos com estabilização da doença, em alguns casos por longos períodos (30+ semanas, por exemplo), sugere que este esquema de tratamento possa afetar favoravelmente a duração da sobrevida em algumas pacientes.

Provavelmente, as características das pacientes portadoras de doença em progressão visceral e apresentando, na maioria dos casos, falha a pelo menos três esquemas quimioterápicos prévios, bem como a limitada atividade do etoposide como droga única neste contexto foram as principais razōes para a ausência de respostas objetivas com esta nova estratégia. Nessas pacientes, pode-se antecipar a presença de uma alta probabilidade de resistência tumoral, o que limita o sucesso de qualquer intervenção quimioterápica ${ }^{1,8}$.

Em resumo, o uso de etoposide em doses orais fracionadas diárias foi bem tolerado, mas não produziu vantagens em termos de respostas objetivas, se comparado à sua forma clássica de administração por via oral em dose única diária. Ainda que algumas pacientes possam ter obtido benefício com essa estratégia, o seu uso não pode ser recomendado na rotina.

Declaração de conflito de interesses: nada a declarar.

\section{REFERÊNCIAS}

1. Jones SE. Considerations in treatment choice for metastatic breast cancer. Breast Cancer. 2008;15(1):35-9.

2. Salvini P, Ripa C, Ginanni V. Metastatic breast cancer: what are the objectives? Tumori. 2000;86(5 Suppl 1):S22-8. 
3. Bergh J, Jönsson PE, Glimelius B, Nygren P; SBU-group. Swedish Council of Technology Assessment in Health Care. A systematic overview of chemotherapy effects in breast cancer. Acta Oncol. 2001;40(2-3):253-81

4. Carrick S, Parker S, Wilcken N, Ghersi D, Marzo M, Simes J. Single agent versus combination chemotherapy for metastatic breast cancer. Cochrane Database Syst Rev. 2005;(2):CD003372.

5. Sledge GW, Neuberg D, Bernardo P, Ingle JN, Martino S, Rowinsky EK, et al. Phase III trial of doxorubicin, paclitaxel, and the combination of doxorubicin and paclitaxel as frontline chemotherapy for metastatic breast cancer: an intergroup trial (E1193). J Clin Oncol. 2003;21(4):588-92. Comment in: J Clin Oncol. 2003;21(4):577-9. J Clin Oncol. 2003;21(4):580-2.

6. Muss HB, Case LD, Richards F 2nd, White DR, Cooper $\mathrm{MR}$, Cruz JM, et al. Interrupted versus continuous chemotherapy in patients with metastatic breast cancer. The Piedmont Oncology Association. N Engl J Med. 1991;325(19):1342-48. Comment in: N Engl J Med. 1991;325(19):1370-1.

7. Falkson G, Gelman R, Pandya K, Osborne CK, Tormey D, Cummings FJ, et al. Eastern Cooperative Oncology Group randomized trials of observation versus maintenance therapy for patients with metastatic breast cancer in complete remission following induction treatment. J Clin Oncol. 1998;16(5):1669-76.

8. Gonzalez-Angulo AM, Morales-Vasquez F, Hortobagyi GN. Overview of resistance to systemic therapy in patients with breast cancer. Adv Exp Med Biol. 2007;608:1-2.

9. Perez EA, Lerzo G, PivotX, Thomas E, Vahdat L, Bosserman L, et al. Efficacy and safety of ixabepilone (BMS-247550) in a phase II study of patients with advanced breast cancer resistant to an anthracycline, a taxane, and capecitabine. J Clin Oncol. 2007;25(23):3407-14. Epub 2007 Jul 2.

10. Miller KD, Wang M, Gralow J, Dickler M, Cobleigh M, Perez EA, et al. Paclitaxel plus bevacizumab versus paclitaxel alone for metastatic breast cancer. N Engl J Med. 2007;357(26):2666-76. Comment in: N Engl J Med. 2008 Apr 10;358(15):1637; author reply 1637-8. N Engl J Med. 2008 Apr 10;358(15):1637; author reply 1637-8.

11. Olver IN. Trastuzumab as the lead monoclonal antibody in advanced breast cancer: choosing which patient and when. Future Oncol. 2008;4(1):125-31.

12. Geyer CE, Forster J, Lindquist D, Chan S, Romieu CG, Pienkowski T, et al. Lapatinib plus capecitabine for HER2positive advanced breast cancer. $\mathrm{N}$ Engl J Med. 2006;355(26):2733-43. Erratum in: N Engl J Med. 2007;356(14):1487. Comment in: N Engl J Med. 2006;355(26):2783-5. N Engl J Med. 2007;356(14):1471; author reply 1471-2. Nat Clin Pract Oncol. 2007;4(7):398-9.

13. Martin M, Lluch A, Casado A, Santabárbara P, Adrover E, Valverde JJ, et al. Clinical activity of oral etoposide in previously treated metastatic breast cancer. J Clin. Oncol. 1994;12(5):986-91. Comment in: J Clin Oncol. 1994;12(10):2235.

14. Pusztai L, Walters RS, Valero V, Theriault RL, Hortobagyi GN. Daily oral etoposide in patients with heavily pretreated metastatic breast cancer. Am J Clin Oncol. 1998;21(5):442-6.

15. Saphner T, Weller EA, Tormey DC, Pandya KJ, Falkson CI, Stewart J, et al. 21-day oral etoposide for metastatic breast cancer: a phase II study and review of the literature. Am J Clin Oncol. 2000;23(3):258-62.

16. Atienza DM, Vogel CL, Trock B, Swain SM. Phase II study of oral etoposide for patients with advanced breast cancer. Cancer. 1995;76(12):2485-90.

17. Neskovic-Konstantinovic ZB, Bosnjak SM, Radulovic SS, Mitrovic LB. Daily oral etoposide in metastatic breast cancer. Anticancer Drugs. 1996;7(5):543-7.

18. Schwartsmann G, Sprinz E, Kromfield M, Kalakun L, Sander E, Prolla G, et al. Clinical and pharmacokinetic study of oral etoposide in patients with AIDS-related Kaposi's sarcoma with no prior exposure to cytotoxic therapy. J Clin Oncol. 1997;15(5):2118-24.

19. Sprinz E, Caldas AP, Mans DR, Cancela A, DiLeone L, Dalla Costa T, et al. Fractionated doses of oral etoposide in the treatment of patients with aids-related kaposi sarcoma: a clinical and pharmacologic study to improve therapeutic index. Am J Clin Oncol. 2001;24(2):177-84.

20. Gregianin LJ, Brunetto AL, Di Leone L, Costa TD, Santos PP, Schwartsmann G. Clinical and pharmacokinetic study of fractionated doses of oral etoposide in pediatric patients with advanced malignancies. Med Sci Monit. 2002;8(9):PI70-7. 


\section{Abstract}

Etoposide is an inhibitor of the nuclear topoisomerase II enzyme, which produces objective tumor responses in about $10 \%$ of patients with metastatic breast cancer failing to standard chemotherapy regimens. Fractionated oral administration of etoposide causes significant increase in tissue drug exposure, leading to a better therapeutic index. In this paper, the outcomes of a Phase II trial of fractionated oral daily doses of etoposide conducted in 20 women with metastatic breast cancer, who progressed following multiple chemotherapy regimens, are described. Eligible patients were those between 18-75 years old, ECOG performance status between 0-2, confirmed histopathological diagnosis of breast cancer, presence of visceral involvement, no vital organs dysfunction and no CNS involvement. A written informed consent was required, in accordance with the local IRB and the Ministry of Health of Brazil. The content of an ampoule for IV use was administered orally, at the dose of 20 $\mathrm{mg} / \mathrm{m} 2$, every eight hours, diluted in a low $\mathrm{pH}$ fluid (orange or grape juice), daily for 14 consecutive days, followed by a 7-day rest. Patients were reviewed every 21 days for toxicity (NCI-CTC criteria), and every 42 days for tumoral response (RECIST criteria). Patients were treated until tumor progression, dose-limiting toxicity or own desire to interrupt the treatment. Twenty patients were included in the trial, and a total of 55 treatment cycles administered with a median of two cycles per patient (1-10) was evaluated. The most common side-effects were nausea $(36 \%)$, vomiting $(24 \%)$, mucositis $(16 \%)$ and neutropenia $(14 \%)$. Febrile neutropenia was documented in one case $(2 \%)$ only. No objective response was documented. However, nine patients showed stable disease (45\%), in some cases with prolonged duration $(30+, 21+$ and 18 weeks). The median duration of stable disease was 15 weeks (9-30+). In summary, this daily fractionated regimen of oral etoposide was well tolerated, producing long-lasting stable disease in some cases. However, no clear advantage in terms of objective tumor responses could be documented, when compared with previous data on its classical single oral administration.

Key words: Etoposide; Breast neoplasms; Neoplasm netastasis 